\title{
Vitamin D improves levels of hormonal, oxidative stress and inflammatory parameters in polycystic ovary syndrome: a meta-analysis study
}

\author{
Jun-Feng Zhao, Bai-Xue Li, Qi Zhang \\ Basic Medical College, Chengdu University of Traditional Chinese Medicine, Chengdu, China \\ Contributions: (I) Conception and design: JF Zhao; (II) Administrative support: BX Li, Q Zhang; (III) Provision of study materials or patients: All \\ authors; (IV) Collection and assembly of data: JF Zhao; (V) Data analysis and interpretation: All authors; (VI) Manuscript writing: All authors; (VII) \\ Final approval of manuscript: All authors. \\ Correspondence to: Qi Zhang. Basic Medical College, Chengdu University of Traditional Chinese Medicine, Chengdu 610072, China. \\ Email: zhangqi@cdutcm.edu.cn.
}

Backgroundk Hyperandrogenism (HA), inflammation, and oxidative damage play key roles in the pathophysiology of polycystic ovary syndrome (PCOS). Whether vitamin D adjuvant therapy improves hormonal, inflammation, and oxidative damage in PCOS patients has aroused widespread interest, but the results are controversial. To evaluate the effect of vitamin D supplementation on hormonal, oxidative stress, and inflammatory parameters in patients with PCOS.

Methods: A literature search was conducted in Medline, the Cochrane Library, EMBASE, and Web of Science for studies related to PCOS and vitamin D supplementation. All reports of randomized controlled trials (RCTs) published before December 2019 were identified. The fixed-effects model or randomeffects model was used to calculate pooled estimates of standardized differences in means (SMD) with $95 \%$ confidence intervals (CI).

Results: A total of 956 identified studies were retrieved, and eighteen RCTs involving 1,060 participants were ultimately included in the current meta-analysis. The pooled results demonstrated that vitamin D supplementation in patients with PCOS resulted in a significant improvement in serum total testosterone (TT), high sensitivity C-reactive protein (hs-CRP), total antioxidant capacity (TAC), and malondialdehyde (MDA). No significant effect on free testosterone (FT), dehydroepiandrosterone sulfate (DHEA-S), sex hormonebinding globulin (SHBG), free androgen index (FAI), nitric oxide (NO), and total glutathione (GSH) levels was found. Subgroup analysis showed that vitamin D supplementation reduced hs-CRP and MDA irrespective of the treatment course, type of vitamin D intervention, supplementation frequency, and dosage. Twelve weeks of vitamin D supplementation improved TT and TAC while low-dose vitamin D supplementation ( $\leq 1,000$ IU/day) improved TT and DHEA-S. Vitamin D co-supplementation reduced TT, FT, and DHEA-S, while a daily supplementation regime improved TT, DHEA-S, and TAC in patients with PCOS.

Conclusions: The current meta-analysis demonstrates that vitamin D supplementation in patients with PCOS resulted in an improvement in the levels of TT, hs-CRP, TAC, and MDA, but did not affect FT, DHEA-S, SHBG, FAI, NO, and GSH levels. Vitamin D co-supplementation, low-dose vitamin D supplementation $(\leq 1,000 \mathrm{IU} /$ day), and daily supplementation frequency appeared to be more conducive to improving hormones, inflammation, and oxidative stress in PCOS patients.

Keywords: Vitamin D; polycystic ovary syndrome (PCOS); hormonal; oxidative stress; inflammation; metaanalysis

Submitted Sep 20, 2020. Accepted for publication Jan 11, 2021.

doi: 10.21037/apm-20-2201

View this article at: http://dx.doi.org/10.21037/apm-20-2201 


\section{Introduction}

Polycystic ovary syndrome (PCOS) is the most common reproductive endocrine disease in women, with a rate of $5-20 \%$ (1). It is characterized by hyperandrogenism (HA), polycystic ovary, ovulation dysfunction, and metabolic disorders. It can also increase the risk of mood disorders, cardiovascular and cerebrovascular diseases, type 2 diabetes, venous thromboembolism, pregnancy diseases, endometrial cancer, and other diseases (1-3). The pathogenesis of PCOS has not been fully elucidated, but current studies have shown that HA, inflammation, and oxidative damage jointly play a vicious cycle in the pathophysiology of the disease (4-8).

Vitamin D deficiency (VDD), which can aggravate HA and insulin resistance, is very common in patients with PCOS, the prevalence rate being as high as $67-85 \%(9-11)$. Numerous animal studies have shown that vitamin D can reduce inflammatory reactions and regulate the physiological balance between oxidation and antioxidants (12-15). However, based on clinical randomized controlled trials (RCTs) (16-33), the effects of vitamin D supplementation on hormone levels, inflammation, and oxidative stress in patients with PCOS are still controversial. The current systematic review and meta-analysis aimed to explore the efficacy of vitamin D supplementation at improving hormone metabolism, inflammation, and oxidative stress levels in patients with PCOS. These studies will play an indispensable role in developing new or adjuvant therapies to treat PCOS in patients. We present the following article in accordance with the PRISMA reporting checklist (available at http://dx.doi.org/10.21037/apm-20-2201).

\section{Methods}

\section{Protocol and registration}

This meta-analysis was conducted following the Preferred Reporting Items for Systematic Reviews and Meta-Analysis (PRISMA) guidelines (34). This study was registered with PROSPERO (Registration No. 42019129803).

\section{Search strategy}

Two authors (Junfeng Zhao and Baixue Li) independently searched databases including Medline, the Cochrane Library, EMBASE, and Web of Science until December 2019. The search was conducted with keywords "polycystic ovary syndrome" or "PCOS" with any of the following keywords: "vitamin D", "ergocalciferols", "cholecalciferol", "25-hydroxyvitamin D2", "hydroxycholecalciferols", "calcifediol", "calcitriol", "24,25-dihydroxyvitamin D3" or "dihydrotachysterol". Review articles and their cited studies were searched manually. There were no restrictions on language, publishing years, and publishing types.

\section{Selection criteria}

All RCTs, double-blinded, single-blinded, or openlabel trials and parallel or cross-over trials with followup time and sample size were included in this metaanalysis. Studies with PCOS women over 18 years old who were administered vitamin $\mathrm{D}$ in any dose and dosage form compared to placebo, positive drug, or blank control were included. The outcome indicators analyzed were levels or changes of serum hormones, oxidative stress, and inflammatory markers before and after vitamin $\mathrm{D}$ or placebo treatment.

Studies that were not RCTs were excluded. Animal studies, in vitro studies, and observational studies were excluded. Studies not assessing primary data, letters to the editor, conference papers, articles only available in abstract form, duplicate publications, and secondary research papers were all excluded.

\section{Data extraction}

Two researchers (Junfeng Zhao and Baixue Li) independently reviewed and screened the literature according to the selection criteria and collected information using a predesigned data extraction table. Any disagreements were resolved through a cross check, discussion, or consultation with the third researcher (Qi Zhang). Where original data was not reported, other information provided in the literature (such as CIS, $\mathrm{P}$ value, and interquartile spacing) was used to calculate the missing data according to the formula provided in the Cochrane manual (35). Where original data was not directly provided in the text or table, it was extracted from the figure. When data was incomplete, the relevant author was contacted, and/or a search was performed on the Clinical Register (clinicaltrials. gov). The following contents were extracted from the selected literature: author, year of publication, study design, sample size, intervention vitamin D dose, course of treatment, average age, body mass index (BMI), serum 25 hydroxyvitamin $\mathrm{D}(25(\mathrm{OH}) \mathrm{D})$.

Before and after the intervention, outcome indicators including total testosterone (TT), free testosterone (FT), 
Table 1 Risk of bias assessment in the included studies

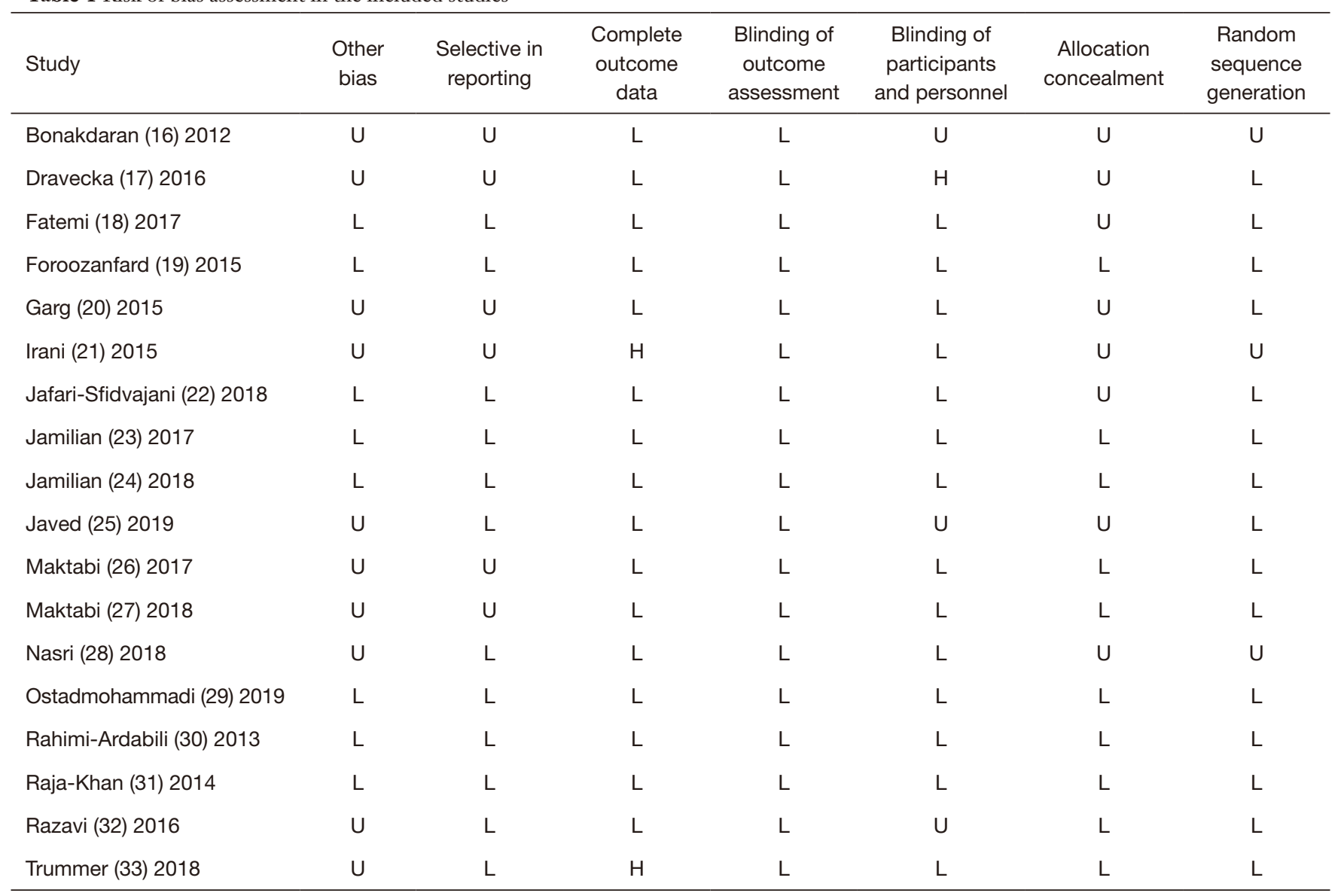

$\mathrm{H}$, high risk; L, low risk; $\mathrm{U}$, unclear risk.

dehydroepiandrosterone sulfate (DHEA-S), sex hormonebinding globulin (SHBG), free androgen index (FAI), high sensitivity C-reactive protein (hs-CRP), nitric oxide (NO), total antioxidant capacity (TAC), malondialdehyde (MDA) and total glutathione (GSH)

\section{Quality assessment}

The quality of the included studies was reviewed according to the Cochrane system evaluation guide manual $(35,36)$. The evaluation contents included random sequence generation, allocation concealment, blinding of outcome assessment, blinding of participants and personnel, incomplete outcome data, selective outcome reporting, and other bias. An answer of "yes" indicated a low risk of bias, an answer of "no" indicated a high risk of bias, and an answer of "unclear" indicated an uncertain risk of bias. The evaluation was conducted independently by two investigators (Junfeng Zhao and Baixue Li), and any disagreements were resolved through discussion or consultation with a third investigator (Qi Zhang). The results of quality assessment are shown in Table 1.

\section{Statistical analysis}

Data was analyzed using Revman 5.3 and Stata 12.0 software and the standardized mean difference (SMD) and 95\% confidence interval (CI) of all outcome indicators were calculated. Q test and $\mathrm{I}^{2}$ test were used to analyze the heterogeneity. In studies with large heterogeneity $(\mathrm{P}<0.10$ or $\mathrm{I}^{2}>50 \%$ ), sensitivity analysis was used to determine the source of heterogeneity and evaluate the stability and reliability of the meta-analysis results. Subgroup analysis was performed examining Vitamin D dosage $(\leq 1,000$ or $>1,000 \mathrm{IU} /$ day), intervention duration (8,12 or 24 weeks), intervention type (vitamin D alone or with a co-supplement) 


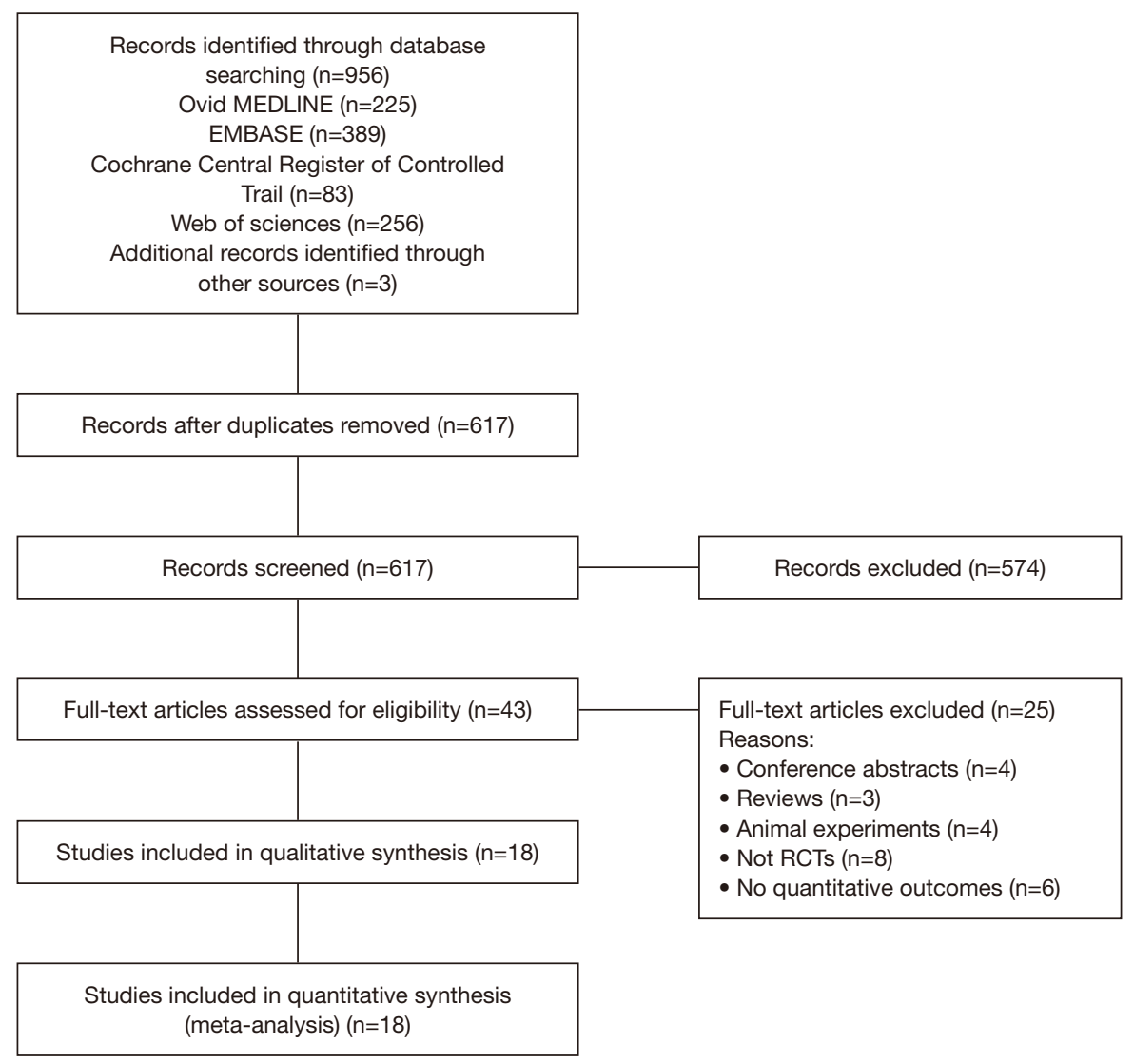

Figure 1 Flow diagram of study selection.

and supplementation manner (daily intake or weekly intake). Funnel plot (Revman 5.3), Egger's and Begg's tests (Stata 12.0 software) were used to determine publication bias (heterogeneity considered present when $\mathrm{P}<0.05$ ). Any publication bias was adjusted using the trim-and-fill method (37) (Stata 12.0 software), with $\mathrm{P}<0.05$ interpreted as statistically different.

\section{Results}

\section{Search results and study characteristics}

The search flow chart is shown in Figure 1. A total of 956 literatures were retrieved and duplicates removed. After literatures were screened through reading titles and abstracts, 43 were selected for full-text reading. Of these, 25 were excluded and eighteen studies were finally included (16-33). Table 2 summarizes the basic characteristics of the included studies. A total of 1,060 subjects were included in the eighteen studies, of which 573 were randomly assigned to the vitamin D group, and the rest were in the control groups. Seventeen control group were placebo-controlled and one was a blank control group. Three studies combined vitamin D with calcium $(19,27,32)$, one study combined vitamin D with vitamin $\mathrm{E}$ (18), one study combined vitamin $\mathrm{D}$ with polyunsaturated fatty acids (PUFA) (24), one study combined vitamin D with evening primrose oil (EPO) (28), and one study combined vitamin D with probiotics (29). In all other studies, patients were supplemented with vitamin $\mathrm{D}$ alone $(16,19,21-23,25,26,30,31,33)$. In the study where metformin was used, it was administered to both the control and study group once a day for 20 weeks (20). The sample size across the studies ranged from 23 to 119 , and the course of treatment ranged from 8 to 24 weeks. The average vitamin D supplement dose ranged from 20 to 12,000 IU/day.

\section{Results of quality assessment}

As shown in Table 1, Among the 18 studies (16-33), 8 
Table 2 Characteristics of included studies

\begin{tabular}{|c|c|c|c|c|c|c|c|c|c|}
\hline \multirow{2}{*}{ Study } & \multirow{2}{*}{$\begin{array}{l}\text { No. of trials }(\mathrm{n}) \text {, } \\
\mathrm{VG} / \mathrm{CG}\end{array}$} & \multirow{2}{*}{ Intervention (dose) } & \multirow{2}{*}{ Control } & \multirow{2}{*}{$\begin{array}{l}\text { Duration } \\
\text { (weeks) }\end{array}$} & \multirow{2}{*}{$\begin{array}{l}\text { Age (year), mean } \pm \mathrm{SD} \text {, } \\
\mathrm{VG} / \mathrm{CG}\end{array}$} & \multirow{2}{*}{$\begin{array}{c}\mathrm{BMI}\left(\mathrm{kg} / \mathrm{m}^{2}\right) \text {, mean } \pm \mathrm{SD} \\
\mathrm{VG} / \mathrm{CG}\end{array}$} & \multicolumn{2}{|c|}{ Vitamin $\mathrm{D}(\mathrm{ng} / \mathrm{mL})$, mean $\pm S D$} & \multirow{2}{*}{ Outcome indicators } \\
\hline & & & & & & & Baseline VG/CG & Final $V G / C G$ & \\
\hline Bonakdaran (16) 2012 & $15 / 16$ & Vitamin D (0.5 $\mu \mathrm{g} /$ day $)$ & Placebo & 12 & $24.7 \pm 3.3 / 25.2 \pm 7.9$ & $24.8 \pm 5.3 / 25.3 \pm 5.1$ & $11.4 \pm 8.2 / 19.9 \pm 16.5$ & $20.1 \pm 16.2^{* * *} / 19.0 \pm 15.3$ & TT, DHEA-S \\
\hline Dravecka (17) 2016 & $11 / 12$ & Vitamin D (1 $\mu \mathrm{g} /$ day $)+$ Metformin & Metformin & 24 & $29.2 \pm 5.4 / 27.6 \pm 4.9$ & $33.1 \pm 7.1 / 29.8 \pm 5.4$ & $22.0 \pm 8.4 / 24.3 \pm 6.8$ & $23.2 \pm 8.2 / 23.5 \pm 6.5$ & $T, F T$, DHEA-S, SHBG, FAI \\
\hline Fatemi (18) 2017 & $44 / 46$ & Vitamin D (50,000 IU/2 week) + Vitamin E & Placebo & 8 & $28.1 \pm 4.2 / 28.1 \pm 3.7$ & $26.5 \pm 3.0 / 26.1 \pm 3.6$ & $7.4 \pm 8.5 / 9.5 \pm 11.9$ & $26 \pm 9.4 / 9.8 \pm 12.6$ & TAC, MDA \\
\hline Foroozanfard (19) 2015 & $26 / 26 / 26$ & $\begin{array}{l}\text { Vitamin D (50,000 IU/week) } \\
\text { Vitamin D + Calcium (50,000 IU/week) }\end{array}$ & Placebo & 8 & NA & NA & NA & NA & hs-CRP, NO, TAC, GSH, MDA \\
\hline Garg (20) 2015 & $15 / 17$ & Vitamin D (4,000 IU/day) + Metformin & $\begin{array}{l}\text { Placebo+ } \\
\text { Metformin }\end{array}$ & 24 & $22.0 \pm 4.6 / 22.8 \pm 4.5$ & $26.8 \pm 4.5 / 26.7 \pm 6.1$ & $7.7 \pm 6.0 / 6.8 \pm 2.4$ & $31.5 \pm 13.8^{\star \star \star} / 6.7 \pm 2.3$ & TT, DHEA-S \\
\hline Irani (21) 2015 & $35 / 18$ & Vitamin D (50,000 IU/week) & Placebo & 8 & $30.5 \pm 1.0 / 29.6 \pm 1.7$ & $30.0 \pm 1.0 / 28.0 \pm 1.6$ & NA & NA & $\Pi \pi, \mathrm{FT}$, DHEA-S, SHBG \\
\hline Jamilian (23) 2017 & $30 / 30 / 30$ & $\begin{array}{l}\text { Low-dose Vitamin D (1,000 IU/day) } \\
\text { High-dose Vitamin D } \\
\text { Placebo (4,000 IU/day) }\end{array}$ & Placebo & 12 & $26.0 \pm 5.0 / 28.0 \pm 5.0 / 25.0 \pm 5.0$ & $33.0 \pm 5.0 / 31.0 \pm 6.0 / 30.0 \pm 6.0$ & $12.6 \pm 3.4 / 12.6 \pm 2.7 / 12.9 \pm 2.4$ & $18.5 \pm 4.9^{* \star *} / 24.6 \pm 3.3^{* * *} / 13.1 \pm 2.5$ & hs-CRP, NO, TAC, GSH, MDA \\
\hline Jamilian (24) 2018 & $30 / 30$ & Vitamin D (50,000 IU/2 week $)+$ PUFA & Placebo & 12 & $26.8 \pm 4.4 / 25.1 \pm 3.7$ & $27.1 \pm 7.0 / 27.4 \pm 3.9$ & $12.1 \pm 3.1 / 12.4 \pm 2.7$ & $21.9 \pm 6.3^{* \star *} / 12.7 \pm 3.2$ & hs-CRP, NO, TAC, GSH, MDA \\
\hline Javed (25) 2019 & $18 / 30$ & Vitamin D (3,200 IU/day) & Placebo & 12 & NA & $35.4 \pm 10.6 / 33.8 \pm 7.2$ & $25.6 \pm 11.4 / 30.9 \pm 11.1$ & $90.4 \pm 19.5^{* * *} / 47.6 \pm 20.5^{* * *}$ & $\pi$, SHBG, FAl, hs-CRP \\
\hline Maktabi (26) 2017 & $35 / 35$ & Vitamin D (25,000 IU/week) & Placebo & 12 & NA & NA & $12.8 \pm 4.5 / 14.5 \pm 5.1$ & $27.5 \pm 9.8^{* \star *} / 14.4 \pm 5.2$ & $\begin{array}{l}\text { TT, FT, DHEA-S, SHBG, FAl, hs- } \\
\text { CRP, NO, TAC, GSH, MDA }\end{array}$ \\
\hline Maktabi (27) 2018 & $30 / 30$ & $\begin{array}{l}\text { Vitamin D (400 IU/day) + Calcium, } \\
\text { magnesium and zinc } \\
\text { Placebo }\end{array}$ & Placebo & 12 & $23.8 \pm 5.7 / 24.8 \pm 4.8$ & $24.0 \pm 3.7 / 25.5 \pm 4.9$ & $10.1 \pm 4.9 / 10.8 \pm 4.6$ & $18.0 \pm 10.3^{\star \star *} / 10.9 \pm 4.5$ & $\begin{array}{l}\text { TT, SHBG, FAl, hs-CRP, NO, TAC, } \\
\text { GSH, MDA }\end{array}$ \\
\hline Ostadmohammadi (29) 2019 & 30/30 & $\begin{array}{l}\text { Vitamin D (25,000 IU/week) + Probiotics } \\
\text { Placebo }\end{array}$ & Placebo & 12 & $24.4 \pm 4.7 / 25.4 \pm 5.1$ & $24.3 \pm 4.2 / 25.1 \pm 4.9$ & $11.1 \pm 4.1 / 12.9 \pm 3.2$ & $24.4 \pm 5.6^{* * *} / 13.3 \pm 2.7$ & $\begin{array}{l}\text { TT, SHBG, hs-CRP, NO, TAC, } \\
\text { GSH, MDA }\end{array}$ \\
\hline Rahimi-Ardabili (30) 2013 & $24 / 26$ & $\begin{array}{l}\text { Vitamin D ( } 50,000 \mathrm{IU} / 20 \text { day }) \\
\text { Placebo }\end{array}$ & Placebo & 12 & $26.8 \pm 4.7 / 27 \pm 3.7$ & $29.1 \pm 4.62 / 28.3 \pm 3.5$ & $6.9 \pm 2.80 / 7.3 \pm 2.9$ & $23.4 \pm 6.14^{* * *} / 8.6 \pm 4.0$ & hs-CRP \\
\hline Raja-Khan (31) 2014 & $13 / 15$ & $\begin{array}{l}\text { Vitamin D (12,000 IU/day) } \\
\text { Placebo }\end{array}$ & Placebo & 12 & $28.2 \pm 5.2 / 28.7 \pm 5.6$ & $37.2 \pm 4.5 / 35.0 \pm 9.8$ & $19.9 \pm 9.4 / 22.2 \pm 6.8$ & $67.3 \pm 28.6^{\star \star \star} / 22.4 \pm 7.0$ & $T T, \mathrm{FT}$, hs-CRP \\
\hline Razavi (32) 2016 & $27 / 27$ & $\begin{array}{l}\text { Vitamin D (400 IU/day) + Calcium } \\
\text { Placebo }\end{array}$ & Placebo & 8 & NA & NA & $14.4 \pm 2.9 / 14.6 \pm 5.5$ & $19.7 \pm 3.1 * * * / 14.5 \pm 6.0$ & $\begin{array}{l}\text { FT, DHEA-S, hs-CRP, NO, TAC, } \\
\text { GSH, MDA }\end{array}$ \\
\hline Trummer (33) 2018 & $78 / 41$ & $\begin{array}{l}\text { Vitamin D (20,000 IU/week) } \\
\text { Placebo }\end{array}$ & Placebo & 24 & $25.4 \pm 4.6 / 27.2 \pm 5.5$ & $20.2 \pm 7.8 / 19.9 \pm 7.3$ & $15.6 \pm 5.3 / 15.6 \pm 5.6$ & $28.7 \pm 6.4^{* *} / 18.2 \pm 9.4^{*}$ & $\pi, \mathrm{FT}$ \\
\hline
\end{tabular}

sensitivity C-reactive protein; NO, nitric oxide; GSH, glutathione; NA, not provided; PUFA, omega-3 polyunsaturated fatty acids; EPO, evening primrose oil. *P<0.05; * * P $<0.01 ; * * * P<0.001$. 


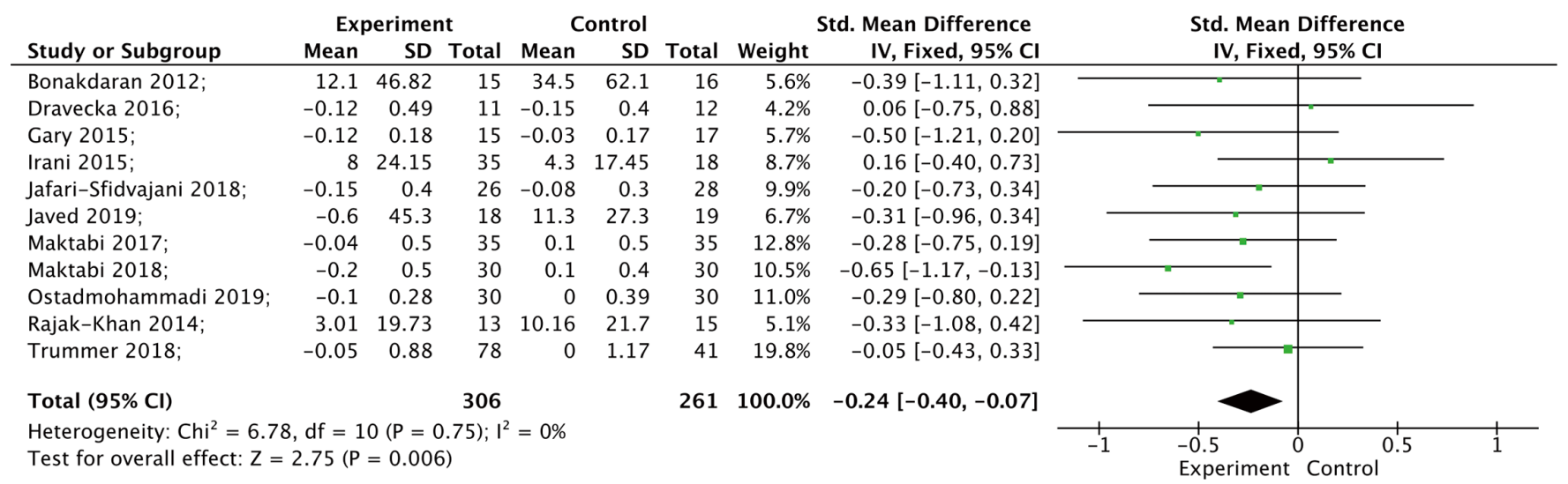

Figure 2 Effects of vitamin D supplementation on total testosterone (TT).

quality assessment projects of 6 studies $(19,23,24,29,30,31)$ were all low-risk, and 7 quality assessment projects of 3 studies $(18,22,33)$ were low-risk, On the whole, the quality of literature included in this meta-analysis was relatively high.

\section{Meta-analysis}

Effects of vitamin D supplementation on testosterone Data on the effect of vitamin D supplementation on TT were reported in eleven studies involving 567 subjects $(16,17,20-22,25-27,29,31,33)$. There was no statistical heterogeneity among the studies $\left(\mathrm{P}=0.75, \mathrm{I}^{2}=0 \%\right)$. Fixed effects model analysis demonstrated that vitamin $\mathrm{D}$ supplementation could significantly reduce serum TT (Figure 2, SMD $=-0.24,95 \% \mathrm{CI}:-0.40,-0.07, \mathrm{P}=0.006$ ). Subgroup analysis showed that 12 weeks of vitamin D supplementation, vitamin D dose less than 1,000 IU/day, vitamin $\mathrm{D}$ combination therapy and daily intake could reduce TT in PCOS patients (Table 3).

\section{Effects of vitamin D supplementation on high sensitivity $\mathrm{C}$-reactive protein}

Nine studies involving 559 subjects examined the effect of vitamin D supplementation on hs-CRP (19,23-27,29,30,32). There was no statistical heterogeneity among the studies $\left(\mathrm{P}=0.11, \mathrm{I}^{2}=36 \%\right)$. Fixed effects model analysis demonstrated that vitamin $\mathrm{D}$ supplementation could significantly reduce serum hs-CRP (Figure 3, SMD $=-0.56,95 \% \mathrm{CI}:-0.73$, $-0.38, \mathrm{P}<0.00001)$. Different course of treatment, type of vitamin $\mathrm{D}$ intervention, frequency and dose of vitamin $\mathrm{D}$ supplementation can reduce hs-CRP in patients with PCOS (Table 3).

\section{Effects of vitamin D supplementation on TAC}

Meta-analysis of nine studies (18,19,23,24,26-29,32) demonstrated that vitamin D supplementation could significantly increase serum TAC levels compared with the control group (Figure 4A, SMD $=0.42,95 \% \mathrm{CI}$ : 0.01, $0.83, \mathrm{P}=0.04)$. As there was high statistical heterogeneity $\left(\mathrm{P}<0.00001, \mathrm{I}^{2}=83 \%\right)$, random effects model analysis was used. Following removal of the two studies with high heterogeneity $(18,28)$, there was less statistical heterogeneity between studies $\left(\mathrm{P}=0.10, \mathrm{I}^{2}=40\right)$. After using the fixed effects model, the difference was still statistically significant (Figure 4B, SMD =0.61, 95\% CI: 0.42, 0.80, $\mathrm{P}<0.00001$ ). Subgroup analysis showed that 12 weeks of vitamin D supplementation and daily administration frequency improved serum TAC in patients with PCOS (Table 3).

\section{Effects of vitamin D supplementation on MDA}

Nine studies reported the effect of vitamin D supplementation on MDA (18,19,23,24,26-29,32), with statistical heterogeneity among the studies $(\mathrm{P}=0.002$, $\left.\mathrm{I}^{2}=63 \%\right)$. The results showed that compared with the control group, vitamin D supplementation treatment could significantly reduce serum MDA (Figure $5 A, \mathrm{SMD}=-0.59$, 95\% CI: $-0.87,-0.32), \mathrm{P}<0.0001)$. After removing the one studiy with high heterogeneity (18), there was no statistical heterogeneity among the studies $\left(\mathrm{P}=0.46, \mathrm{I}^{2}=0 \%\right)$. In the fixed effects model analysis, the results demonstrated that vitamin D supplementation significantly reduced serum MDA (Figure 5B, SMD $=-0.72,95 \%$ CI: $-0.90,-0.54$, $\mathrm{P}<0.00001)$. Subgroup analysis revealed that different courses of treatment, type of vitamin D intervention, frequency and dose of vitamin D supplementation could reduce MDA in PCOS patients (Table 3). 


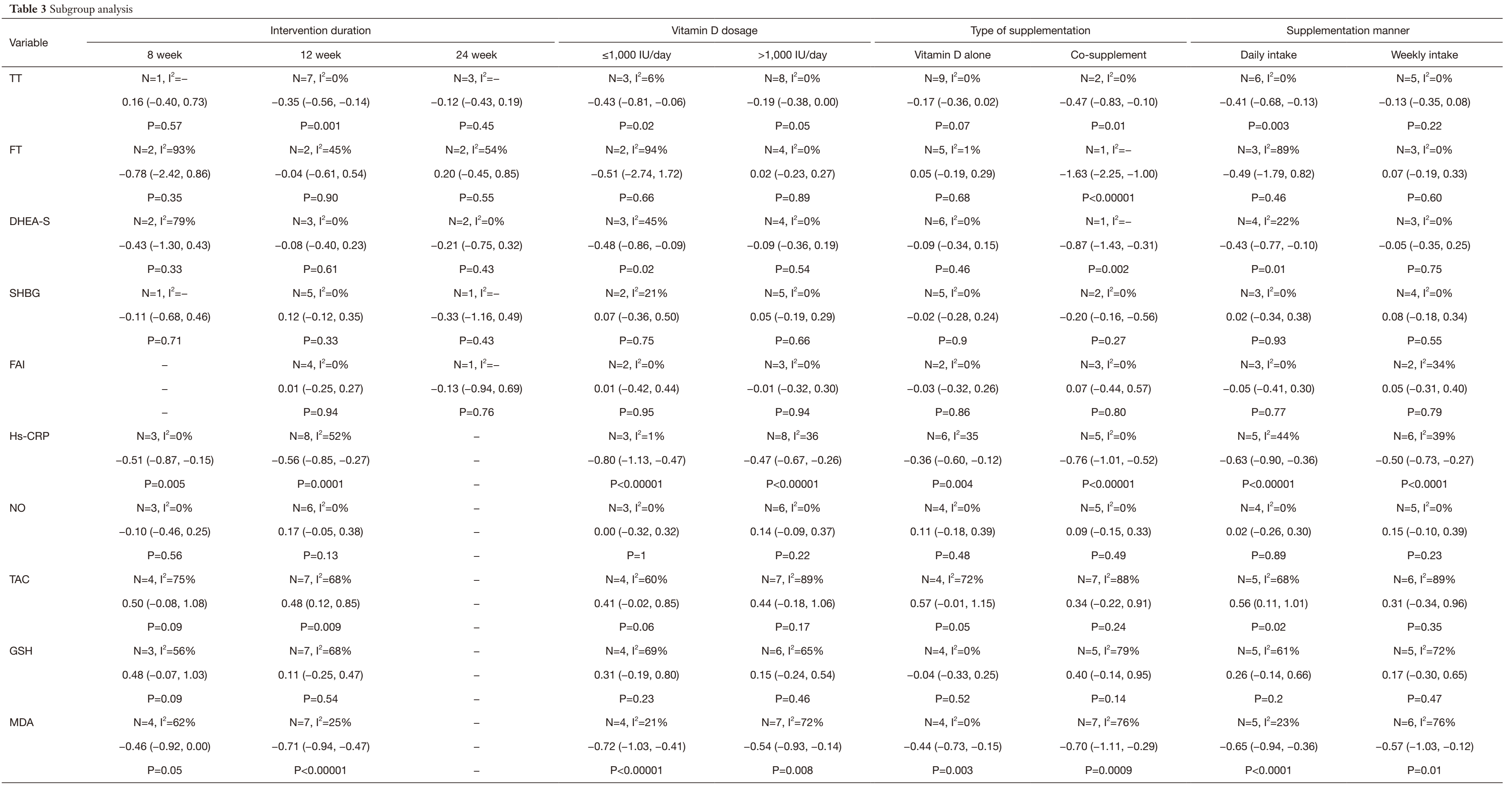

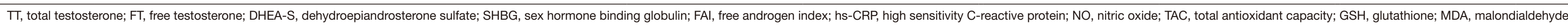




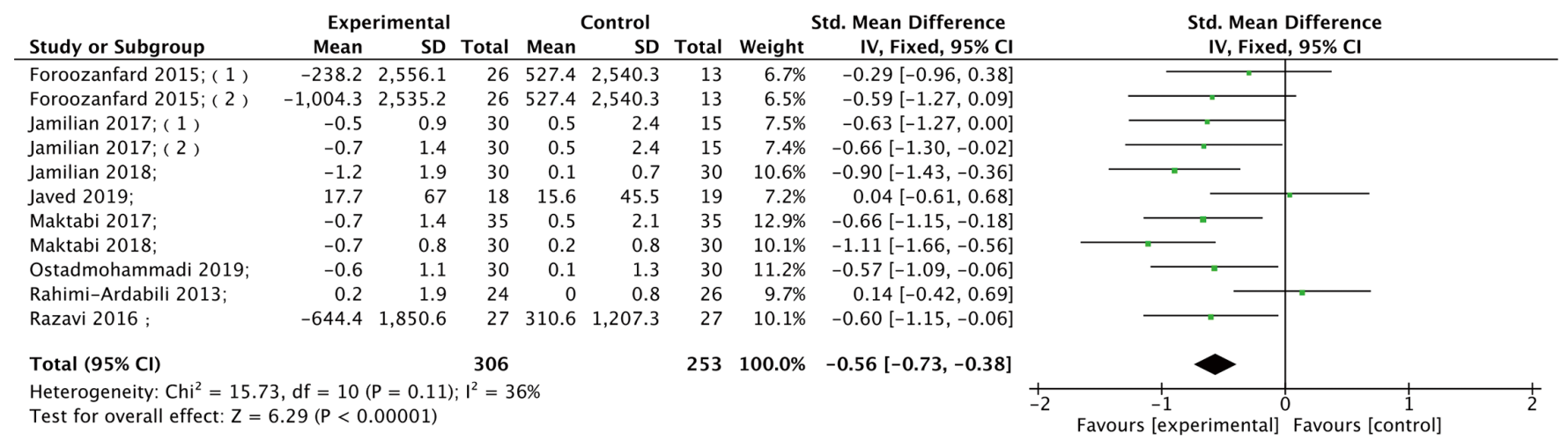

Figure 3 Effects of vitamin D supplementation on high-sensitivity C-reactive protein (hs-CRP).

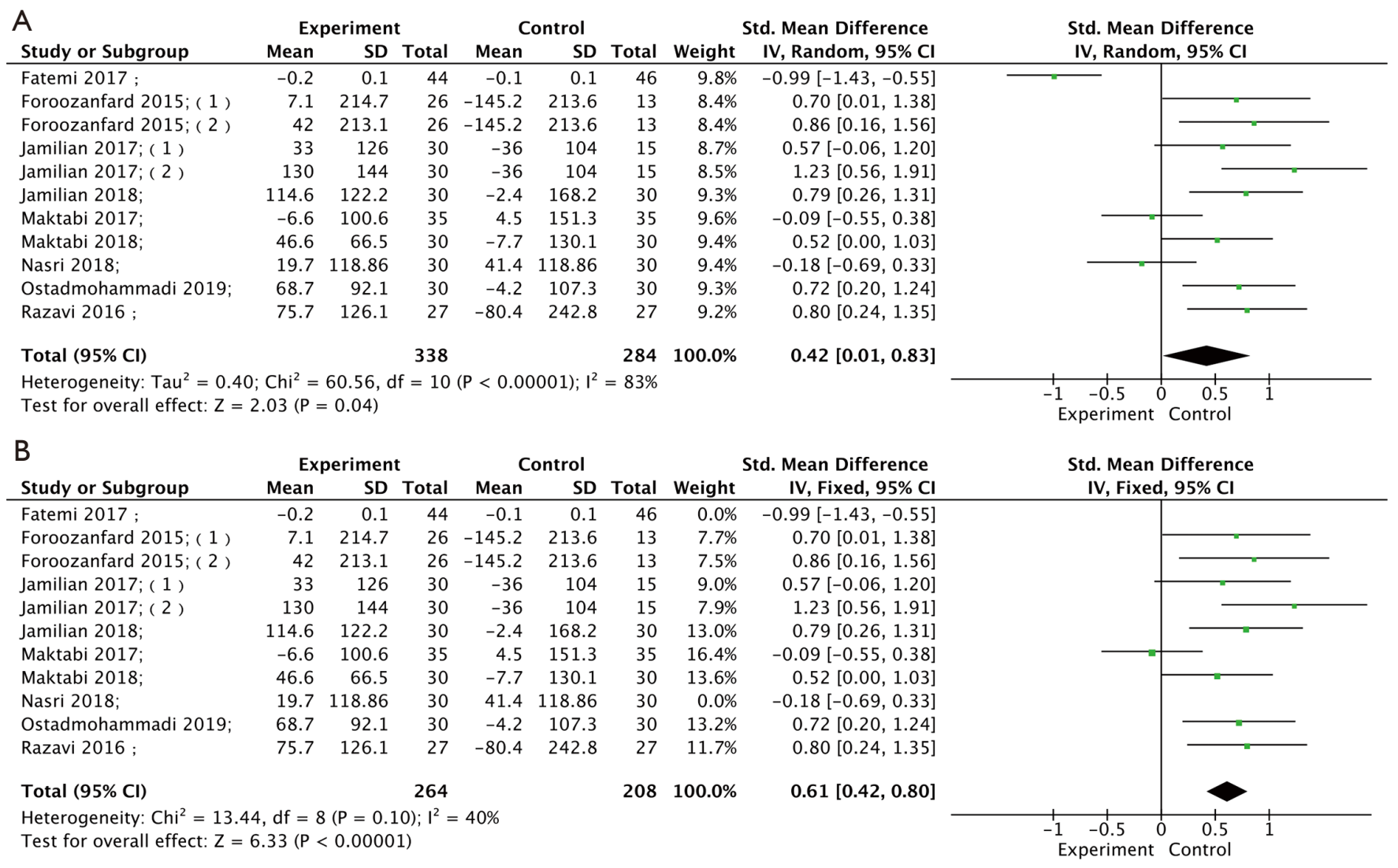

Figure 4 Effects of vitamin D on total antioxidant capacity (TAC). (A) Effects of vitamin D supplementation on TAC and (B) re-analyzed data of the effect of vitamin D supplementation on TAC.

\section{Effects of vitamin D supplementation on levels of} FT, dehydroepiandrosterone sulphate, sex hormone binding globulin, FAI, NO and GSH

As shown in Table 4, the effect of vitamin D on FT was evaluated in six studies $(17,21,26,31-33)$. There was no significant difference in FT between the vitamin D treatment and the control group $(\mathrm{P}=0.47)$. Subgroup analysis showed that vitamin $\mathrm{D}$ combined with calcium supplementation could reduce FT in PCOS patients (Table 3). The effect of vitamin D supplementation on 


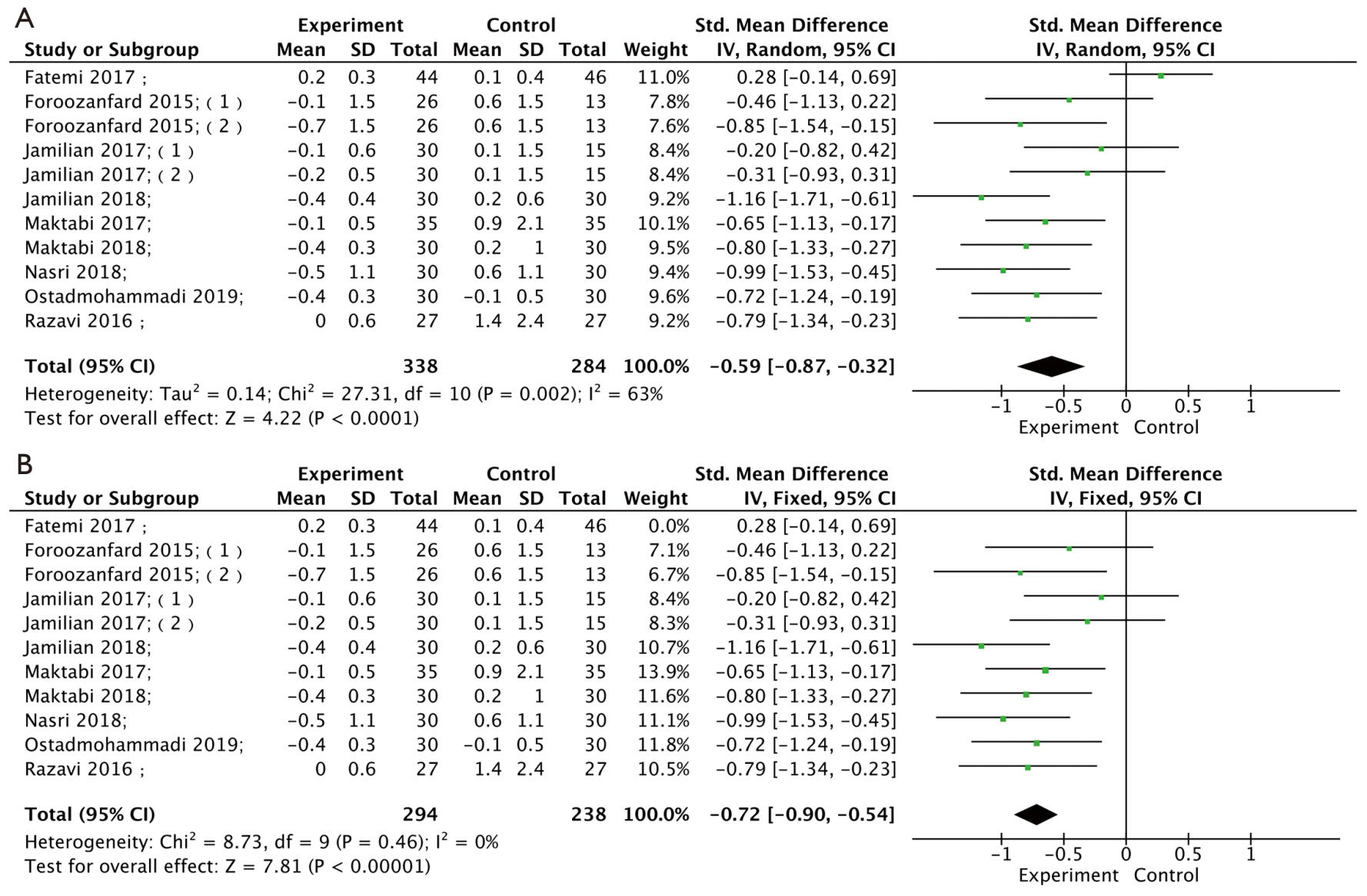

Figure 5 Effects of vitamin D supplementation on malondialdehyde (MDA). (A) Effects of vitamin D supplementation on MDA and (B) reanalyzed data of the effect of vitamin D supplementation on MDA.

DHEA-S was reported in seven studies $(16,17,20,22,26,32)$, and the effect on SHBG was also reported in seven studies $(17,21,22,25,27,29)$. The fixed effects model analysis showed that compared with the control group, serum DHEA-S sulfate treatment demonstrated a downward trend, but there was no statistical significance $(\mathrm{P}=0.06)$. Vitamin D supplementation had no significant effect on improving SHBG $(\mathrm{P}=0.59)$. Subgroup analysis showed that vitamin $\mathrm{D}$ dose less than 1,000 IU/day, vitamin D combined with supplement therapy and daily administration frequency could reduce the level of DHEA-S in PCOS patients (Table 3). Five studies reported the effect of vitamin D supplementation on FAI $(17,22,25,26,27)$. The combined results demonstrated that vitamin $\mathrm{D}$ supplementation had no significant effect on FAI ( $\mathrm{P}=0.99)$. Seven studies evaluated the effect of vitamin D on NO $(19,23,24,26,27,29,32)$. Again, vitamin D supplementation had no significant effect on NO levels $(\mathrm{P}=0.32)$. Eight studies reported the effect of vitamin D supplementation on GSH $(19,23,24,26-29,32)$. The random effects model analysis showed no significant difference in GSH between the vitamin $\mathrm{D}$ treatment group and the control group $(\mathrm{P}=0.16)$.

\section{Heterogeneity analysis}

There is some heterogeneity in the analysis of FT, TAC, GSH and MDA. The sensitivity analysis of the sources demonstrated that the heterogeneity mainly derived from the studies by Fatemi (18), Nasri (28) and Razavi (32). Therefore, the data was re-analyzed after excluding these major heterogeneity studies. The results of FT, TAC, GSH and MDA analysis before and after the removal of major heterogeneity sources did not change (TAC: Figure 4B; MDA: Figure 5B; FT and GSH: Table 4). In the above three studies vitamin $\mathrm{D}$ treatment was combined with a co- 
Table 4 Effects of vitamin D supplementation on FT, DHEA-S, SHBG, FAI, NO and GSH levels

\begin{tabular}{|c|c|c|c|c|c|c|c|}
\hline Variable & $\begin{array}{c}\text { Model for } \\
\text { meta-analysis }\end{array}$ & Subject (n) & No. of trials & $\begin{array}{c}\text { Effect size }(95 \% \mathrm{Cl}) \\
\mathrm{mg} / \mathrm{dL}\end{array}$ & $P$ value & $\mathrm{I}^{2}(\%)$ & Q-statistics $(P)$ \\
\hline \multicolumn{8}{|c|}{ Total results } \\
\hline DHEA-S & FE & 7 & 317 & $-0.22(-0.44,0.01)$ & 0.06 & 13 & 0.330 \\
\hline FAl & FE & 5 & 244 & $0.00(-0.25,0.25)$ & 0.99 & 0 & 0.680 \\
\hline NO & FE & 7 & 472 & $0.09(-0.09,0.28)$ & 0.32 & 0 & 0.950 \\
\hline GSH & $\mathrm{RE}$ & 8 & 532 & $0.21(-0.09,0.51)$ & 0.16 & 65 & 0.002 \\
\hline \multicolumn{8}{|c|}{ Results after removing the main heterogeneity sources } \\
\hline
\end{tabular}

FE, fixed effect model; RE, random effect model; FT, free testosterone; DHEA-S, dehydroepiandrosterone sulfate; SHBG, sex hormone binding globulin; FAl, free androgen index; No, nitric oxide; GSH, glutathione.

supplement $(18,28,32)$. The vitamin D dose administered fluctuated greatly from 400 to 7,143 IU/day. Also, subgroup analysis demonstrated that the heterogeneity of most subgroups was significantly reduced (Table 3). Therefore, we infer that the heterogeneity may come from different vitamin $\mathrm{D}$ supplements, treatment courses, intervention types and frequency. Also, differences in age, sex ratio, patient health status (such as baseline vitamin D levels, comorbidities, etc.) and study quality may also be additional sources of heterogeneity.

\section{Publication bias}

We evaluated the publication bias of the outcome indicators with statistical significance. The funnel plot results are shown in Figure 6. Egger's and Begg's tests showed that vitamin $\mathrm{D}$ supplementation had no publication bias on TT (Begg's: $\mathrm{P}=0.533$ and Egger's: $\mathrm{P}=0.270$ ), hs-CRP (Begg's: $\mathrm{P}=0.276$ and Egger's: $\mathrm{P}=0.360$ ) and MDA (Begg's: $\mathrm{P}=0.640$ and Egger's: $\mathrm{P}=0.094)$. However, publication bias was found on TAC (Begg's: $\mathrm{P}=0.020$ ). After adjusting for the bias by the trim-and-fill method, the total results of TAC did not change (before: $\mathrm{SMD}=0.42,95 \% \mathrm{CI}: 0.01,0.83, \mathrm{P}=0.04$; after: $\mathrm{SMD}=0.64,95 \%$ CI: $0.39,0.89, \mathrm{P}<0.00001)$.

\section{Discussion}

This meta-analysis of eighteen RCTs studies involving
1,060 subjects was conducted to assess the effects of vitamin D supplementation on hormones, oxidative stress, and inflammatory markers in women with PCOS. The current research demonstrated that vitamin $\mathrm{D}$ supplementation could improve TT, hs-CRP, TAC, and MDA in PCOS patients, but there was no significant difference in FT, DHEA-S, SHBG, FAI, NO, and GSH levels. Subgroup analysis showed that different courses of treatment, type of vitamin $\mathrm{D}$ intervention, frequency, and dose of vitamin D supplementation could reduce hs-CRP and MDA in PCOS patients. Vitamin D supplementation for 12 weeks can improve TT and TAC, low-dose vitamin D $(\leq 1,000 \mathrm{IU} /$ day $)$ can improve TT and DHEA-S. Vitamin D combined with a co-supplement, can improve TT, FT, and DHEA-S, while daily administration frequency can improve TT, DHEA-S, and TAC.

\section{Effect of vitamin D supplementation on hormone}

HA is an important pathophysiological factor in PCOS. High androgen levels in local and circulating ovaries can cause ovarian dysfunction, resulting in abnormal ovulation, irregular menstruation, and even amenorrhea (1). Also, hyperandrogen-like changes, such as hirsutism, acne, and skin seborrhea, are some of the characteristics of PCOS. Our results suggest that vitamin D supplementation improves TT levels in women with PCOS. This is consistent with the results of Pal and colleagues (38). 

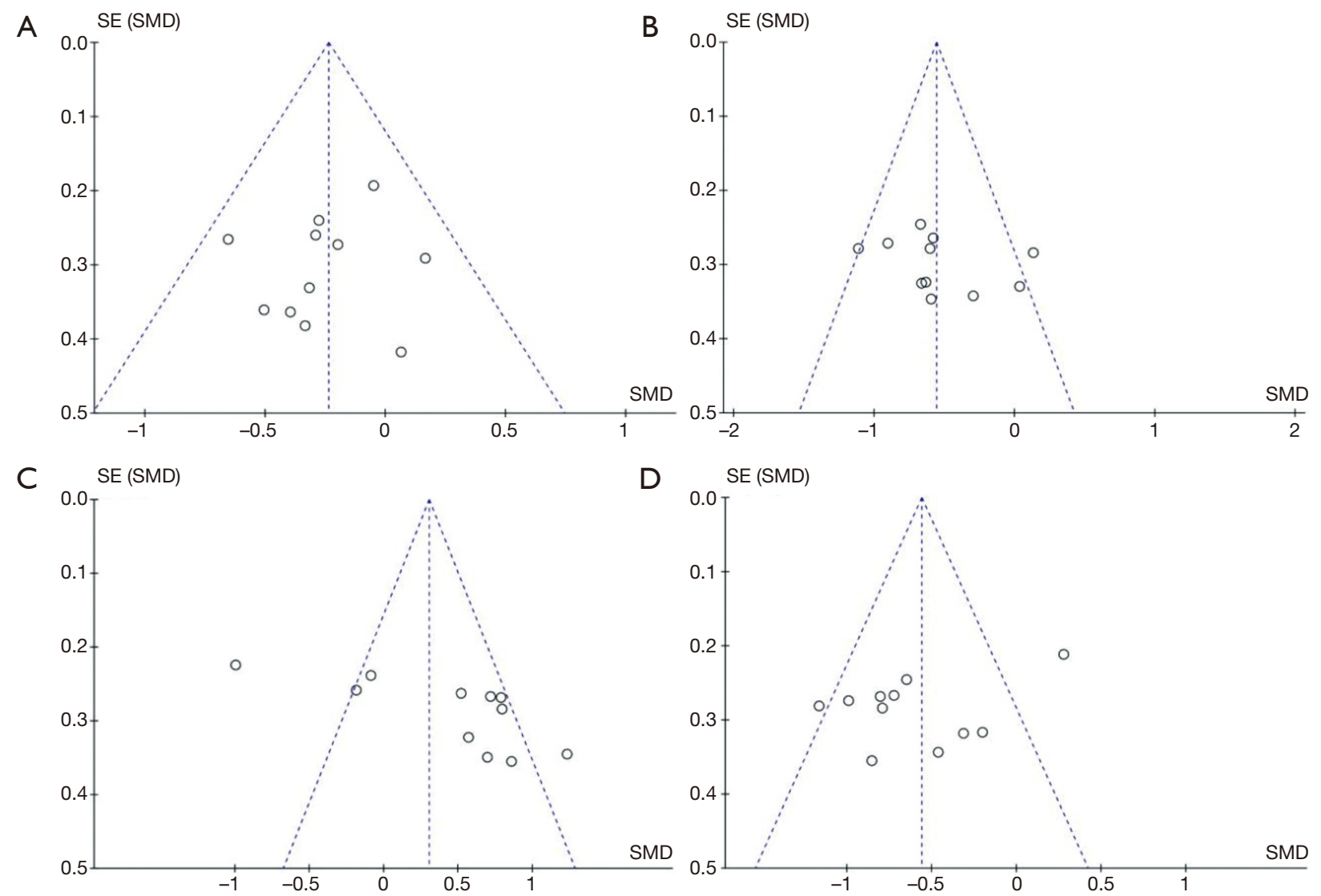

Figure 6 Funnel plot of standard error by standard differences in the means of plasma (A) total testosterone (TT); (B) high-sensitivity C-reactive protein (hs-CRP); (C) total antioxidant capacity (TAC); and (D) malondialdehyde (MDA).

In their single arm open label trial, twelve overweight women with VDD who received vitamin $\mathrm{D}$ and calcium for three months showed decreased TT $(\mathrm{P}=0.036)$ compared with baseline. Also, a study by Firouzabadi and colleagues demonstrated that vitamin D combined with calcium supplement therapy can reduce weight, promote follicular maturation, and improve menstrual disorders and hyperandrogen related symptoms in PCOS patients (39). Observational studies demonstrated that $25(\mathrm{OH}) \mathrm{D}$ levels in PCOS patients were negatively correlated with TT, DHEA-S, and SHBG $(40,41)$. Also, Muscogiuri and colleagues showed that vitamin $\mathrm{D}$ might reduce PCOS androgen by participating in the synthesis of steroids (11).

\section{Effects of vitamin D supplementation on inflammation and oxidative stress}

Insulin resistance and hyperinsulinemia are important characteristics of PCOS patients. Chronic inflammatory reactions and, in turn, inflammatory mediators play an important role in the occurrence and development of insulin resistance. These inflammatory mediators directly affect the phosphorylation and activation process of IRS1 and IRS-2, thereby blocking the transmission of insulin biological signals and causing insulin resistance (42). A key transcription factor regulating the inflammatory response is the nuclear transcription factor kappa-B (NFкB) (42). Rasheed and others have shown that vitamin $\mathrm{D}$ can inhibit NF-кB, reducing the production of free radicals and pro-inflammatory cytokines (43). Our metaanalysis showed that serum hs-CRP levels decreased after vitamin D supplementation in patients with PCOS. This was consistent with the study by Ngo and colleagues (44), which found a negative correlation between serum 25(OH)D level and hs-CRP. Compared with non-PCOS patients, the level of oxidative stress in PCOS patients is elevated, and the proportion of oxidants is higher than that of antioxidants (45). Hyperinsulinemia can stimulate 
the production of reactive oxygen species (ROS) in local tissues and cells, leading to oxidative stress $(46,47)$. Also, PCOS induced ovulation treatment can cause an increase in oxidative stress (46). Inflammation and oxidative damage play key roles in the pathophysiology of diseases $(4,5)$. As MDA is a product of local tissue reaction with ROS, it can be used to reflect the production of ROS. In ROS generation, the antioxidant enzyme (superoxide dismutase, SOD) in the body is continuously consumed, leading to a decrease in TAC levels, and both MDA and TAC are related to the degree of oxidative stress reaction $(48,49)$. Our meta-analysis demonstrated that vitamin D supplementation could improve TAC and MDA levels in PCOS patients. This is consistent with the results of Asemi and colleagues (50), showing that the markers of oxidative stress were significantly improved after six weeks of vitamin $\mathrm{D}$ and calcium supplementation in patients with gestational diabetes mellitus. Cavalcante and others found that after taking 200,000 IU vitamin D for 4 weeks, TAC increased, but MDA concentration was not affected (51). Studies by Jain and colleagues suggested that vitamin D may improve oxidative stress by increasing intracellular GSH levels and reducing ROS and proinflammatory factors (52).

\section{Subgroup analysis}

Subgroup analysis found that vitamin D combined with supplementation appeared to be more effective than vitamin D alone in PCOS patients. Foroozanfard and colleagues treated 104 overweight PCOS women with VDD with $50,000 \mathrm{IU}$ vitamin D per week and 1,000 $\mathrm{mg}$ calcium daily for 8 weeks (19). They observed that compared with placebo, vitamin $\mathrm{D}$ combined with calcium significantly improved hs-CRP, MDA, TAC, and GSH. Tabesh and others found that compared with vitamin $\mathrm{D}$ alone, co-supplementation with calcium can improve the chronic inflammation of diabetic patients with VDD by reducing the concentration of interleukin- 6 and tumor necrosis factor- $\alpha$ (53). Among the included studies in this meta-analysis, three studies coadministered vitamin D with calcium $(19,27,32)$, one with vitamin $\mathrm{E}$ (18), one with unsaturated fatty acids (24), one with grass oil (28), and one with probiotics (29). However, due to the small number of relevant studies, it could not be determined, which co-supplement combination with vitamin $\mathrm{D}$ was most effective. Further RCT studies and mesh metaanalysis will be required.

In the subgroup of vitamin $\mathrm{D}$ supplementation alone, TT also showed a downward trend, but it was not statistically significant $(\mathrm{P}=0.07)$. It cannot be ruled out that the sample size included in the subgroup analysis was too small.

Our subgroup analysis also demonstrated that low-dose vitamin D supplementation ( $\leq 1,000 \mathrm{IU} /$ day) was sufficient to produce significant benefits in the treatment of PCOS. This finding is consistent with the meta-analysis results of Xue (54) and Lagowska (55). They demonstrated that lowdose vitamin D supplementation was sufficient to reduce triglycerides and (homeostasis model assessment of insulin resistance, HOMA-IR) levels in PCOS patients, while high-dose vitamin $\mathrm{D}$ supplementation did not result in any benefit (54,55). Also, Sanders and colleagues noted that high-dose vitamin D (>500,000 IU) might increase fracture risk and cause tolerance problems (56). But it cannot be excluded that low-dose vitamin D supplementation may result in better patient compliance which may influence the results of those studies. Daily administration may be more beneficial to the treatment of PCOS than weekly or fortnightly frequency. Increased frequency of administration may lead to improved absorption of vitamin $D$ in the intestinal tract. A smaller dose of administered daily may also improve tolerance. Most of the studies in this metaanalysis had an intervention time of 12 weeks, with only a few studies using 8 or 24 weeks. Therefore, due to the small sample size, we could not draw any conclusions on the effect of intervention time on vitamin $\mathrm{D}$ supplementation in PCOS patients.

Considering the limitations of the whole subgroup analysis, such as the differences in treatment drugs and the difference is administration time, further high-quality prospective studies are required to verify our results.

In this current meta-analysis, English RCTs were selected through mainstream medical databases using a detailed retrieval strategy. The latest research was included and collected. Eighteen studies were RCT studies, seventeen of which were placebo-controlled, with high quality and reliable data. However, this study was limited by the large variations in vitamin $\mathrm{D}$ supplementation doses, the relatively small sample size, and the variations in cosupplement drugs and administration regimes employed. All these factors likely played a role in increasing the heterogeneity of this meta-analysis.

\section{Conclusions}

This meta-analysis data demonstrated that vitamin D supplementation could improve TT, hs-CRP, TAC, and MDA in PCOS patients. Vitamin D combined 
with supplementary therapy, vitamin D dose less than 1,000 IU/day, and daily dose frequency appeared to be more conducive to improving hormone, inflammation, and oxidative stress in patients with PCOS. Considering the limitations of the current study, further large-scale and high-quality RCT studies are still necessary to better assess the impact of vitamin D supplementation on women with PCOS.

\section{Acknowledgments}

Funding: This study was supported by The National Natural Science Fund of China (No. 81774400).

\section{Footnote}

Reporting Checklist: The authors have completed the PRISMA reporting checklist. Available at http://dx.doi. org/10.21037/apm-20-2201

Conflicts of Interest: All authors have completed the ICMJE uniform disclosure form (available at http://dx.doi. org/10.21037/apm-20-2201). The authors have no conflicts of interest to declare.

Ethical Statement: The authors are accountable for all aspects of the work in ensuring that questions related to the accuracy or integrity of any part of the work are appropriately investigated and resolved.

Open Access Statement: This is an Open Access article distributed in accordance with the Creative Commons Attribution-NonCommercial-NoDerivs 4.0 International License (CC BY-NC-ND 4.0), which permits the noncommercial replication and distribution of the article with the strict proviso that no changes or edits are made and the original work is properly cited (including links to both the formal publication through the relevant DOI and the license). See: https://creativecommons.org/licenses/by-nc-nd/4.0/.

\section{References}

1. Azziz R, Carmina E, Chen Z, et al. Polycystic ovary syndrome. Nat Rev Dis Primers 2016;2:16057.

2. Ruan X, Li M, Mueck AO. Why does Polycystic Ovary Syndrome (PCOS) Need Long-term Management? Curr Pharm Des 2018;24:4685-92.

3. Anagnostis P, Tarlatzis BC, Kauffman RP. Polycystic ovarian syndrome (PCOS): Long-term metabolic consequences. Metabolism 2018;86:33-43.

4. Walters KA, Gilchrist RB, Ledger WL, et al. New Perspectives on the Pathogenesis of PCOS: Neuroendocrine Origins. Trends Endocrinol Metab 2018;29:841-52.

5. Artimani T, Karimi J, Mehdizaeh M, et al. Evaluation of pro-oxidant-antioxidant balance $(\mathrm{PAB})$ and its association with inflammatory cytokines in polycystic ovary syndrome (PCOS). Gynecol Endocrinol 2018;34:148-52.

6. Caldwell ASL, Edwards MC, Desai R, et al. Neuroendocrine androgen action is a key extraovarian mediator in the development of polycystic ovary syndrome. Proc Natl Acad Sci U S A 2017;114:E3334-43.

7. Shorakae S, Teede H, De Courten B, et al. The Emerging Role of Chronic Low-Grade Inflammation in the Pathophysiology of Polycystic Ovary Syndrome. Semin Reprod Med 2015;33:257-69.

8. Ho CW, Chen HH, Hsieh MC, et al. Hashimoto's thyroiditis might increase polycystic ovary syndrome and associated comorbidities risks in Asia. Ann Transl Med 2020;8:684.

9. Thomson RL, Spedding S, Buckley JD. Vitamin D in the aetiology and management of polycystic ovary syndrome. Clin Endocrinol (Oxf) 2012;77:343-50.

10. Maidana P, Fritzler A, Mpcarbe Y, et al. Association Between Vitamin D and Adrenal Parameters with Metabolic and Inflammatory Markers in Polycystic Ovary Syndrome. Sci Rep 2019;9:3968.

11. Muscogiuri G, Alteri B, De Angelis C, et al. Shedding new light on female fertility: The role of vitamin D. Rev Endocr Metab Disord 2017;18:273-83.

12. Manna P, Achari A E, Jain S K. Vitamin D supplementation inhibits oxidative stress and upregulate SIRT1/AMPK/GLUT4 cascade in high glucose-treated 3T3L1 adipocytes and in adipose tissue of high fat diet-fed diabetic mice. Arch Biochem Biophys 2017;615:22-34.

13. Hajiluian G, Abbasalizad Farhangi M, Nameni G, et al. Oxidative stress-induced cognitive impairment in obesity can be reversed by vitamin D administration in rats. Nutr Neurosci 2018;21:744-52.

14. Masszi G, Benko R, Csibi N, et al. Endothelial relaxation mechanisms and nitrative stress are partly restored by Vitamin D3 therapy in a rat model of polycystic ovary syndrome. Life sciences 2013;93:133-8.

15. Zhang M, Lin L, Xu C, et al. VDR Agonist Prevents Diabetic Endothelial Dysfunction through Inhibition of Prolyl Isomerase-1-Mediated Mitochondrial Oxidative Stress and Inflammation. Oxid Med Cell Longev. 
2018;2018:1714896.

16. Bonakdaran S, Mazloom Khorasani Z, Davachi B, et al. The effects of calcitriol on improvement of insulin resistance, ovulation and comparison with metformin therapy in PCOS patients: a randomized placebo- controlled clinical trial. Iran J Reprod Med 2012;10:465-72.

17. Dravecká I, Figurová J, Javorský M, et al. The effect of alfacalcidiol and metformin on phenotype manifestations in women with polycystic ovary syndrome - a preliminary study. Physiol Res 2016;65:815-22.

18. Fatemi F, Mohammadzadeh A, Sadeghi MR, et al. Role of vitamin $\mathrm{E}$ and D3 supplementation in Intra-Cytoplasmic Sperm Injection outcomes of women with polycystic ovarian syndrome: A double blinded randomized placebocontrolled trial. Clin Nutr ESPEN 2017;18:23-30.

19. Foroozanfard F, Jamilian M, Bahmani F, et al. Calcium plus vitamin D supplementation influences biomarkers of inflammation and oxidative stress in overweight and vitaminD-deficient women with polycystic ovary syndrome: a randomized double-blind placebo-controlled clinical trial. Clin Endocrinol (Oxf) 2015;83:888-94.

20. Garg G, Kachhawa G, Ramot R, et al. Effect of vitamin $\mathrm{D}$ supplementation on insulin kinetics and cardiovascular risk factors in polycystic ovarian syndrome: a pilot study. Endocr Connect 2015;4:108-16.

21. Irani M, Seifer DB, Grazi RV, et al. Vitamin D Supplementation Decreases TGF-beta1 Bioavailability in PCOS: A Randomized Placebo-Controlled Trial. J Clin Endocrinol Metab 2015;100:4307-14.

22. Jafari-Sfidvajani S, Ahangari R, HozooriI M, et al. The effect of vitamin D supplementation in combination with low-calorie diet on anthropometric indices and androgen hormones in women with polycystic ovary syndrome: a double-blind, randomized, placebo-controlled trial. J Endocrinol Invest 2018;41:597-607.

23. Jamilian M, Foroozanfard F, Rahmani E, et al. Effect of Two Different Doses of Vitamin D Supplementation on Metabolic Profiles of Insulin-Resistant Patients with Polycystic Ovary Syndrome. Nutrients 2017;9:1280.

24. Jamilian M, Samimi M, Mirhosseini N, et al. The influences of vitamin D and omega-3 co-supplementation on clinical, metabolic and genetic parameters in women with polycystic ovary syndrome. J Affect Disord 2018;238:32-8.

25. Javed Z, Papageorgiou M, Deshmukh H, et al. A Randomized, Controlled Trial of Vitamin D Supplementation on Cardiovascular Risk Factors, Hormones, and Liver Markers in Women with Polycystic
Ovary Syndrome. Nutrients 2019;11:188.

26. Maktabi M, Chamani M, Asemi Z. The Effects of Vitamin D Supplementation on Metabolic Status of Patients with Polycystic Ovary Syndrome: A Randomized, DoubleBlind, Placebo-Controlled Trial. Horm Metab Res 2017;49:493-8.

27. Maktabi M, Jamilian M, Asemi Z. Magnesium-ZincCalcium-Vitamin D Co-supplementation Improves Hormonal Profiles, Biomarkers of Inflammation and Oxidative Stress in Women with Polycystic Ovary Syndrome: a Randomized, Double-Blind, PlaceboControlled Trial. Biol Trace Elem Res 2018;182:21-8.

28. Nasri K, Akrami S, Rahimi M, et al. The effects of vitamin $\mathrm{D}$ and evening primrose oil co-supplementation on lipid profiles and biomarkers of oxidative stress in vitamin D-deficient women with polycystic ovary syndrome: A randomized, double-blind, placebo-controlled trial. Endocr Res 2018;43:1-10.

29. Ostadmohammadi V, Jamilian M, Bahani F, et al. Vitamin $\mathrm{D}$ and probiotic co-supplementation affects mental health, hormonal, inflammatory and oxidative stress parameters in women with polycystic ovary syndrome. J Ovarian Res 2019;12:5.

30. Rahimi-Ardabili H, Pourghassem Gargari B, Farzadi L. Effects of vitamin D on cardiovascular disease risk factors in polycystic ovary syndrome women with vitamin D deficiency. J Endocrinol Invest 2013;36:28-32.

31. Raja-Khan N, Shah J, Stetter C M, et al. High-dose vitamin D supplementation and measures of insulin sensitivity in polycystic ovary syndrome: a randomized, controlled pilot trial. Fertil Steril 2014;101:1740-6.

32. Razavi M, Jamillian M, Karamali M, et al. The Effects of Vitamin D-K-Calcium Co-Supplementation on Endocrine, Inflammation, and Oxidative Stress Biomarkers in VitaminD-Deficient Women with Polycystic Ovary Syndrome:A Randomized, Double-Blind,PlaceboControlled Trial. Horm Metab Res 2016;48:446-51.

33. Trummer C, Schwez V, Kollmann M, et al. Effects of vitamin $\mathrm{D}$ supplementation on metabolic and endocrine parameters in PCOS: a randomized-controlled trial. Eur J Nutr 2019;58:2019-28.

34. Shamseer L, Moher D, Clarke M, et al. Preferred reporting items for systematic review and meta-analysis protocols (PRISMA-P) 2015: elaboration and explanation. BMJ 2015;350:g7647.

35. Julian PTH, Sally G, editors. Cochrane Handbook for Systematic Reviews of Interventions: Cochrane Handbook for Systematic Reviews of Interventions Version 5.1.0; 
(cited 2011 Mar 20). Available online: http://handbook. cochrane.org

36. Jørgensen L, Paludan-Müller AS, Laursen DR, et al. Evaluation of the Cochrane tool for assessing risk of bias in randomized clinical trials: overview of published comments and analysis of user practice in Cochrane and non-Cochrane reviews. Syst Rev 2016;5:80.

37. Duval S, Tweedie R. Trim and fill: A simple funnel-plotbased method of testing and adjusting for publication bias in meta-analysis. Biometrics 2000;56:455-63.

38. Pal L, Berry A, Coraluzzi L, et al. Therapeutic implications of vitamin D and calcium in overweight women with polycystic ovary syndrome. Gynecol Endocrinol 2012;28:965-8.

39. Firouzabadi R, Aflatoonian A, Modarrsesi S, et al. Therapeutic effects of calcium \&vitamin D supplementation in women with PCOS. Complement Ther Clin Pract 2012;18:85-8.

40. Yildizhan R, Kurdoglu M, Adali E, et al. Serum 25-hydroxyvitamin D concentrations in obese and nonobese women with polycystic ovary syndrome. Arch Gynecol Obstet 2009;280:559-63.

41. Velija-Ašimi Z. Evaluation of the association of vitamin D deficiency with gonadotropins and sex hormone in obese and non-obese women with polycystic ovary syndrome. Med Glas (Zenica) 2014;11:170-6.

42. Zuo T, Zhu M, Xu W, et al. Iridoids with Genipin Stem Nucleus Inhibit Lipopolysaccharide-Induced Inflammation and Oxidative Stress by Blocking the NF-kappaB Pathway in Polycystic Ovary Syndrome. Cell Physiol Biochem 2017;43:1855-65.

43. Al-Rasheed NM, Al-Rasheed NM, Bassiouni YA, et al. Vitamin D attenuates pro-inflammatory TNF-alpha cytokine expression by inhibiting NF-small ka, CyrillicB/ p65 signaling in hypertrophied rat hearts. J Physiol Biochem 2015;71:289-99.

44. Ngo DT, Sverdlov AL, Mcneil JJ, et al. Does vitamin D modulate asymmetric dimethylarginine and C-reactive protein concentrations?. Am J Med 2010;123:335-41.

45. Murri M, Luque-Ramirez M, Insenser M, et al. Circulating markers of oxidative stress and polycystic ovary syndrome (PCOS): a systematic review and meta-analysis. Hum Reprod Update 2013;19:268-88.

46. Sumithra NU, Lakshmi RL, Leela Menon N, et al. Evaluation of Oxidative Stress and hsCRP in Polycystic Ovarian Syndrome in a Tertiary Care Hospital. Indian J Clin Biochem 2015;30:161-6.

47. Hyderali BN, Mala K. Oxidative stress and cardiovascular complications in polycystic ovarian syndrome. Eur J Obstet Gynecol Reprod Biol 2015;191:15-22.

48. Victor VM, Rovira-Llopis S, Banuls C, et al. Insulin Resistance in PCOS Patients Enhances Oxidative Stress and Leukocyte Adhesion: Role of Myeloperoxidase. PloS one 2016;11:e0151960.

49. Pekel A, Gonenc A, Turhan NO, et al. Changes of sFas and sFasL, oxidative stress markers in serum and follicular fluid of patients undergoing IVF. J Assist Reprod Genet 2015;32:233-41.

50. Asemi Z, Karamali M, Esmaillzadeh A. Effects of calciumvitamin D co-supplementation on glycaemic control, inflammation and oxidative stress in gestational diabetes: a randomized placebo-controlled trial. Diabetologia 2014;57:1798-806.

51. de Medeiros Cavalcante IG, Silva AS, Costa MJ, et al. Effect of vitamin D3 supplementation and influence of BsmI polymorphism of the VDR gene of the inflammatory profile and oxidative stress in elderly women with vitamin D insufficiency: Vitamin D3 megadose reduces inflammatory markers. Exp Gerontol 2015;66:10-6.

52. Jain SK, Michinski D. Vitamin D upregulates glutamate cysteine ligase and glutathione reductase, and GSH formation, and decreases ROS and MCP-1 and IL-8 secretion in high-glucose exposed U937 monocytes. Biochem Biophys Res Commun 2013;437:7-11.

53. Tabesh M, Azadbakht L, Faghihimani E, et al. Calciumvitamin D cosupplementation influences circulating inflammatory biomarkers and adipocytokines in vitamin D-insufficient diabetics: a randomized controlled clinical trial. J Clin Endocrinol Metab 2014;99:E2485-93.

54. Xue Y, Xu P, Xue K, et al. Effect of vitamin D on biochemical parameters in polycystic ovary syndrome women: a meta-analysis. Arch Gynecol Obstet 2017;295:487-96.

55. Łagowska K, Bajerska J, Jamka M. The Role of Vitamin D Oral Supplementation in Insulin Resistance in Women with Polycystic Ovary Syndrome: A Systematic Review and Meta-Analysis of Randomized Controlled Trials. Nutrients. 2018;10:1637.

56. Sanders KM, Nicholson GC, Ebeling PR. Is high dose vitamin D harmful?. Calcif Tissue Int 2013;92:191-206.

Cite this article as: Zhao JF, Li BX, Zhang Q. Vitamin D improves levels of hormonal, oxidative stress and inflammatory parameters in polycystic ovary syndrome: a meta-analysis study. Ann Palliat Med 2021;10(1):169-183. doi: 10.21037/apm-20-2201 